\title{
On Linear Difference Equations for Which the Global Periodicity Implies the Existence of an Equilibrium
}

\author{
István Gyóri and László Horváth \\ Department of Mathematics, University of Pannonia, Egyetem Utca 10, Veszprém 8200, Hungary \\ Correspondence should be addressed to István Győri; gyori@almos.uni-pannon.hu \\ Received 5 September 2013; Revised 4 November 2013; Accepted 18 November 2013 \\ Academic Editor: Agacik Zafer
}

Copyright ( 2013 I. Győri and L. Horváth. This is an open access article distributed under the Creative Commons Attribution License, which permits unrestricted use, distribution, and reproduction in any medium, provided the original work is properly cited.

It is proved that any first-order globally periodic linear inhomogeneous autonomous difference equation defined by a linear operator with closed range in a Banach space has an equilibrium. This result is extended for higher order linear inhomogeneous system in a real or complex Euclidean space. The work was highly motivated by the early works of Smith $(1934,1941)$ and the papers of Kister (1961) and Bas (2011).

\section{Introduction}

Let $X$ be a set and let $p$ be a positive integer. It is said that the transformation $T: X \rightarrow X$ is $p$-periodic if

$$
T^{p}:=T \circ \cdots \circ T=i d_{X},
$$

where $i d_{X}$ is the identical function on $X$ and $p$ is the least positive integer with this property. It follows from (1) that $T$ is a bijection. If there is a topology on $X$ and $T$ is continuous, then (1) implies that $T$ is a homeomorphism.

The following question was posed by Smith (see [1]): does any continuous periodic transformation of a Euclidean $n$ space always admit a fixed point? Smith knew that the answer is true if the period $p$ of the transformation is a prime number (see [2]) or a power of a prime number (see [1]). Moreover, Smith was able to answer the question affirmatively when $n \leq 3$ and for suitably regular transformations, when $n=4$. But it was shown by Kister (see [3]) that there exist periodic transformations of a Euclidean space without fixed points. Kister's example is based on the results in the paper [4].

Special periodic transformations can be derived from difference equations.

Consider the sth order difference equation:

$$
x(n)=h(x(n-1), \ldots, x(n-s)) \quad n \geq 0,
$$

where,
(G) $s$ is a positive integer, $X$ is a set, and $h: X^{s} \rightarrow X$.

It is clear that the solutions of (2) are uniquely determined by their initial values:

$$
x(n)=\varphi(n), \quad-s \leq n \leq-1,
$$

where $\varphi(n) \in X$. The unique solution of (2) and (3) is denoted by $x(\varphi)=(x(\varphi)(n))_{n \geq-s}$, where $\varphi:=(\varphi(-s), \ldots, \varphi(-1))^{T} \epsilon$ $X^{s}$. (2).

We give some basic definitions about the periodicity of

Definition 1. Assume (G).

(a) A sequence $v=(v(n))_{n \geq-s}$ in $X$ is called periodic if there is a positive integer $p$ such that $v$ is $p$-periodic, which means that $v(n+p)=v(n)$ for all $n \geq-s$.

(b) We say that (2) is globally periodic if there is a positive integer $p \geq s$ for which the equation is globally $p$ periodic; that is, every solution of it is $p$-periodic.

(c) We say that (2) is globally $p$-periodic with prime period $p$ if it is globally $p$-periodic and $p$ is the least positive integer with this property. 
It is easy to see that (2) is globally $p$-periodic with prime period $p$ if and only if the transformation $T: X^{s} \rightarrow X^{s}$ defined by

$$
\begin{aligned}
& T\left(x_{-s}, \ldots, x_{-1}\right) \\
& \quad:=\left(x_{-s+1}, \ldots, x_{-2}, h\left(x_{-s}, \ldots, x_{-1}\right)\right)
\end{aligned}
$$

is $p$-periodic.

About periodicity of general difference equations, see [5, 6]. Periodicity of linear difference equations is considered in [7].

We recall that the solution $(x(n))_{n \geq-s}$ of (2) is a steady state solution if $x(n)=v(n \geq-s)$, where $v \in X$ is an equilibrium of (2); that is, $v$ obeys

$$
v=h(v, \ldots, v) .
$$

It is obvious that $v \in X$ is an equilibrium of (2) exactly if $(v, \ldots, v)$ is a fixed point of the transformation $T$ given in (4).

Even if there is a metric on $X$ and $h$ is continuous, it is still an open problem to determine whether (2) has or not an equilibrium point, or equivalently, the transformation (4) has a fixed point, if (2) is globally periodic.

In this paper we solve this problem for some linear equations.

Let $\mathbb{K}$ stand for either the field of real numbers $\mathbb{R}$ or the field of complex numbers $\mathbb{C}$. Throughout this paper, the term vector space in which the scalar field is not explicitly mentioned will refer to a vector space over $\mathbb{R}$ or over $\mathbb{C}$.

Consider the sth order inhomogeneous linear difference equation:

$$
x(n)=\sum_{i=1}^{s} L_{i}(x(n-i))+b, \quad n \geq 0,
$$

where,

(A) $s$ is a positive integer, $X$ is a vector space, $L_{i}: X \rightarrow X$ is a linear transformation $(1 \leq i \leq s)$, and $b \in X$ is a vector.

The sth order homogeneous linear difference equation associated (6) is

$$
x(n)=\sum_{i=1}^{s} L_{i}(x(n-i)), \quad n \geq 0 .
$$

Clearly, if that (6) is globally p-periodic, the difference of any two solutions of it is also $p$-periodic. On the other hand, the general solution of the inhomogeneous equation (6) can be written as the sum of the general solution of the homogeneous equation (7) and an arbitrarily fixed particular solution of the inhomogeneous equation. Thus the global $p$-periodicity of the inhomogeneous equation implies the global $p$-periodicity of the related homogeneous equation. One can easily see that the opposite statement is also true if the inhomogeneous equation has a steady state solution which is obviously $p$-periodic for any $p \geq 1$.

From this we conclude the following.
Conclusion. If (6) has an equilibrium, then (6) and (7) both behave in the same way regarding the global periodicity; that is, they both are globally periodic or both are not globally periodic.

The crux in the application of the above self-evident statement is that not all autonomous inhomogeneous linear difference equations have an equilibrium. But this crux is eliminated by the main theorems of this work in two special cases of (6).

In the first result $X$ is finite dimensional.

Theorem 2. Consider the system of the sth order inhomogeneous linear difference equations:

$$
x(n)=\sum_{i=1}^{s} A_{i} x(n-i)+b, \quad n \geq 0,
$$

where,

(B) $s$ is a positive integer, $A_{i} \in \mathbb{K}^{d \times d}(1 \leq i \leq s)$ are matrices, and $b \in \mathbb{K}^{d}$ is vector.

If (8) is globally periodic, then it has an equilibrium.

Let $X$ be a vector space. $I$ and $O$ mean the identity and the zero operator on $X$, respectively. If $L: X \rightarrow X$ is a linear transformation, we define the kernel and the image of $L$ in the usual way:

$$
\begin{gathered}
\operatorname{ker}(L):=\{x \in X \mid L(x)=0\}, \\
\operatorname{im}(L):=\{L(x) \mid x \in X\} .
\end{gathered}
$$

In the next result first-order equations are investigated.

Theorem 3. Consider the first order inhomogeneous linear difference equation:

$$
x(n)=L(x(n-1))+b, \quad n \geq 0,
$$

where,

(C) $L$ is a bounded linear operator of the Banach space $X$ into itself such that $\operatorname{im}(I-L)$ is closed and $b \in X$ is a vector.

If (10) is globally periodic, then it has an equilibrium.

\section{Existence of an Equilibrium in an Abstract First-Order Inhomogeneous Linear Equation}

In this section we prove Theorem 3.

First, we need the following lemma about global periodicity.

Lemma 4. Consider the first order inhomogeneous linear difference equation:

$$
x(n)=L(x(n-1))+b, \quad n \geq 0,
$$


where,

(D) $X$ is a vector space, $L: X \rightarrow X$ is a linear transformation, and $b \in X$ is a vector.

Let $p$ be a positive integer. Equation (11) is globally $p$ periodic if and only if

$$
L^{p}=I, \quad \sum_{i=0}^{p-1} L^{i}(b)=0 .
$$

Proof. It is easy to check that (11) is globally p-periodic if and only if

$$
\left(L^{p}-I\right) \varphi+\sum_{i=0}^{p-1} L^{i}(b)=0
$$

for every $\varphi \in X$, but this condition and (12) are equivalent.

Remark 5. (a) Condition (12) is equivalent to

$$
\left(\sum_{i=0}^{p-1} L^{i}\right)(I-L)=O, \quad b \in \operatorname{ker}\left(\sum_{i=0}^{p-1} L^{i}\right) .
$$

The first part of (14) implies that

$$
\operatorname{im}(I-L) \subset \operatorname{ker}\left(\sum_{i=0}^{p-1} L^{i}\right) .
$$

Since (11) has an equilibrium point exactly if the linear equation

$$
(I-L) x=b
$$

has a solution, it follows from the previous establishments that the following two assertions are equivalent. Let $p$ be a positive integer.

(i) If (11) is globally $p$-periodic, then it has an equilibrium.

(ii) If $L^{p}=I$, then

$$
\operatorname{im}(I-L)=\operatorname{ker}\left(\sum_{i=0}^{p-1} L^{i}\right) .
$$

(b) $L^{p}=I$ implies that $L$ is invertible. If $I-L$ is also invertible, then (16) obviously has a solution (or (17) holds), and therefore the only interesting case is when $I-L$ is not invertible.

We can see that if (11) is globally periodic, then the problem of the existence or nonexistence of an equilibrium leads to a pure linear algebraic problem.

Problem. Let $X$ be a vector space and let $L: X \rightarrow X$ be a linear transformation such that $L^{p}=I$ for some integer $p \geq 2$. Either prove that

$$
\operatorname{im}(I-L)=\operatorname{ker}\left(\sum_{i=0}^{p-1} L^{i}\right)
$$

or give an example when $\operatorname{im}(I-L)$ is a proper subset of

$$
\operatorname{ker}\left(\sum_{i=0}^{p-1} L^{i}\right)
$$

If $L$ is a linear operator of the Banach space $X$ into itself such that $\operatorname{im}(I-L)$ is closed, then Theorem 3 shows that (18) holds.

Henceforth we need some notations (see [8]).

Definition 6. Let $X$ be a Banach space.

(a) $X^{*}$ means its dual space, and let $(w, u)$ denote the value of the functional $w \in X^{*}$ at $u \in X$. For a bounded linear operator $L$ of $X$ into itself, $L^{*}: X^{*} \rightarrow$ $X^{*}$ denotes its adjoint operator.

(b) Suppose that $M$ is a subspace of $X$ and $N$ is a subspace of $X^{*}$. Their annihilators are defined as follows:

$$
\begin{gathered}
M^{\perp}:=\left\{w \in X^{*} \mid(w, u)=0, u \in M\right\}, \\
{ }^{\perp} N:=\{u \in X \mid(w, u)=0, w \in N\} .
\end{gathered}
$$

In the proof of Theorem 3 the following result will be used, which is related to the Fredholm alternative (see [9]).

Lemma 7. Let $X$ be a Banach space and let $L$ be a bounded linear operator of $X$ into itself such that $\operatorname{im}(I-L)$ is closed. The equation $(I-L) x=b$ is solvable for given $b \in X$ if and only if $b \epsilon^{\perp}\left(\operatorname{ker}\left(I-L^{*}\right)\right)$.

Proof. It is well known (see [8]) that

$$
{ }^{\perp}\left(\operatorname{ker}\left(I-L^{*}\right)\right)={ }^{\perp}\left(\operatorname{im}(I-L)^{\perp}\right),
$$

and ${ }^{\perp}\left(\operatorname{im}(I-L)^{\perp}\right)$ is the norm closure of $\operatorname{im}(I-L)$ in $X$. Since $\operatorname{im}(I-L)$ is closed,

$$
{ }^{\perp}\left(\operatorname{ker}\left(I-L^{*}\right)\right)=\operatorname{im}(I-L),
$$

which gives the result.

Remark 8. If $X$ is finite dimensional, then $\operatorname{im}(I-L)$ is closed, since every subspace of $X$ is closed. In this case Lemma 7 is exactly the Fredholm alternative.

Proof of Theorem 3. We can obviously suppose that $p \geq 2$.

Equation (10) has an equilibrium point exactly if the linear equation

$$
(I-L) x=b
$$

has a solution. By Lemma 7, it is enough to show that

$$
b \in^{\perp}\left(\operatorname{ker}\left(I-L^{*}\right)\right) \text {. }
$$

To prove (24), assume that

$$
w \in \operatorname{ker}\left(I-L^{*}\right) .
$$


Recalling Lemma 4, we have

$$
\begin{aligned}
(w, b) & =\left(L^{*}(w),-\sum_{i=1}^{p-1} L^{i}(b)\right) \\
& =-\left(w, \sum_{i=1}^{p-1} L^{i+1}(b)\right) \\
& =-\left(w, b+\sum_{i=2}^{p-1} L^{i}(b)\right) \\
& =-\langle w, b\rangle-\left(\sum_{i=2}^{p-1}\left(L^{*}\right)^{i} w, b\right) .
\end{aligned}
$$

$w=L^{*}(w)$ gives $w=\left(L^{*}\right)^{i}(w)$. Consequently,

$$
(w, b)=-(p-1)(w, b)
$$

which means that $(w, b)=0$.

The proof is complete.

By Remark 8, we have the following.

Corollary 9. Consider the first order inhomogeneous linear difference equation:

$$
x(n)=L(x(n-1))+b, \quad n \geq 0,
$$

where $L$ is a linear operator of the finite dimensional space $X$ into itself and $b \in X$ is a vector. If (28) is globally periodic, then it has an equilibrium.

We illustrate by an example that the conditions involved in Theorem 3 can be satisfied and not only the finite dimensional case.

Example 10. Let $B([0,1])$ be the Banach space of bounded scalar-valued functions on $[0,1]$, with the supremum norm

$$
\|f\|_{\infty}:=\sup \{|f(t)| \mid t \in[0,1]\} .
$$

Define the function $\alpha \in B([0,1])$ by

$$
\alpha(t):= \begin{cases}1, & \text { if } t \text { is rational } \\ -1, & \text { if } t \text { is irrational, }\end{cases}
$$

and introduce the following bounded linear operator $L$ on $B([0,1])$ :

$$
L(f):=\alpha f, \quad f \in B([0,1]) .
$$

Then $L^{2}=I, I-L$ is not invertible (by Remark 5 (b), this is an interesting case), and

$$
\operatorname{im}(I-L)=\{g \in B([0,1]) \mid g(t)=0 \text { if } t \text { is rational }\}
$$

is a closed subspace of $B([0,1])$.

It is easy to see that equation

$$
\begin{gathered}
x(n)=L(x(n-1))+b, \\
x, b \in B([0,1]), n \geq 0,
\end{gathered}
$$

or equivalently, for every $t \in[0,1]$

$$
\begin{array}{r}
x(n)(t)=\alpha(t) x(n-1)(t)+b(t), \\
x, b \in B([0,1]), n \geq 0,
\end{array}
$$

is globally 2-periodic if and only if $b \in \operatorname{im}(I-L)$, and in this case it has the equlibrium point $(1 / 2) b$.

The previous example can be extended if the scalars are the complex numbers. Let $p \geq 3$ be an integer, and define the function $\alpha \in B([0,1])$ by

$$
\alpha(t):= \begin{cases}1, & \text { if } t \text { is rational } \\ \varepsilon_{p}, & \text { if } t \text { is irrational, }\end{cases}
$$

where

$$
\varepsilon_{p}:=e^{(2 \pi / p) i}
$$

is a primitive $p$ th root of unity. Then $L^{p}=I$; equation

$$
\begin{aligned}
& x(n)=L(x(n-1)), \\
& x \in B([0,1]), n \geq 0,
\end{aligned}
$$

is globally $p$-periodic, and it has solutions with prime period $p$.

\section{The Proof of Theorem 2}

We will use the following notations.

Definition 11. Let $m \geq 1$ be an integer.

(a) $B V^{m, d}$ will mean the $m d$-dimensional real vector space of block vectors with entries in $\mathbb{K}^{d}$.

(b) The real vector space of $m \times m$ block matrices with entries in $\mathbb{K}^{d \times d}$ will be denoted by $B M^{m, d}\left(B M^{m, d}\right.$ and $\mathbb{K}^{m d \times m d}$ can be treated as being identical).

(c) The zero matrix and the identity matrix in $\mathbb{K}^{d \times d}$ are denoted by $O_{d}$ and $I_{d}$, respectively.

Let $(x(n))_{n \geq-s}$ be a given sequence in $\mathbb{K}^{d}$. Then for any fixed $n \geq 0$ we introduce an $s d$-dimensional state vector:

$$
\mathbf{x}_{n}=\left(\mathbf{x}_{n}(-s), \ldots, \mathbf{x}_{n}(-1)\right)^{T} \in B V^{s, d},
$$

defined by $\mathbf{x}_{n}(i):=x(n+i)(-s \leq i \leq-1)$.

As it is well known (see [10]), by using the state vector notation, (8) may be written as an $s d$-dimensional system of first order difference equations.

Lemma 12. For any $\varphi=(\varphi(-s), \ldots, \varphi(-1))^{T} \in B V^{s, d}, x(\varphi)=$ $(x(\varphi)(n))_{n \geq-s}$ is the solution of (8) and (3) exactly if

$$
\left(\mathbf{x}_{k}(\varphi)\right)_{k \geq 1}=\left(\left(\mathbf{x}_{k}(\boldsymbol{\varphi})(-s), \ldots, \mathbf{x}_{k}(\boldsymbol{\varphi})(-1)\right)^{T}\right)_{k \geq 1}
$$

is the solution of

$$
\begin{gathered}
\mathbf{x}_{k}=\mathscr{C} \mathbf{x}_{k-1}+\mathscr{B}, \quad k \geq 1, \\
\mathbf{x}_{0}=\boldsymbol{\varphi},
\end{gathered}
$$


where the companion matrix $\mathscr{C} \in B M_{\mathbb{K}}^{s, d}$ and the block vector $\mathscr{B}$ can be written in the forms

$$
\begin{gathered}
\mathscr{C}=\left(\begin{array}{ccccc}
O_{d} & I_{d} & O_{d} & \ldots & O_{d} \\
O_{d} & O_{d} & I_{d} & \ldots & O_{d} \\
\vdots & \vdots & \vdots & \ddots & \vdots \\
O_{d} & O_{d} & O_{d} & \ldots & I_{d} \\
A_{s} & A_{s-1} & A_{s-2} & \ldots & A_{1}
\end{array}\right), \\
\mathscr{B}=\left(\begin{array}{c}
0 \\
0 \\
\vdots \\
0 \\
b
\end{array}\right)
\end{gathered}
$$

Another companion matrix is developed in [11].

There is a one-to-one correspondence between the global periodicity of (8) and that of (40) and also between equilibrium of (8) and that of (40).

Lemma 13. (a) Let $p \geq s$ be an integer. Equation (8) is globally $p$-periodic if and only if (40) is also globally p-periodic.

(b) $c \in \mathbb{K}^{d}$ is an equilibrium of (8) exactly if $\mathbf{c}=$ $(c, \ldots, c)^{T} \in B V^{s, d}$ is an equilibrium of $(40)$.

Now we prove the first main result.

Proof of Theorem 2. We can apply Theorem 3 and Lemma 13.

\section{Acknowledgments}

The authors would like to thank the referees for helpful comments and suggestions which improved the presentation of the paper. This paper is supported by the Hungarian National Foundations for Scientific Research Grant no. K101217.

\section{References}

[1] P. A. Smith, "Fixed-points theorems for periodic transformations," American Journal of Mathematics, vol. 63, pp. 1-8, 1941.

[2] P. A. Smith, "A theorem on fixed points for periodic transformations," Annals of Mathematics, vol. 35, pp. 572-578, 1934.

[3] J. M. Kister, "Examples of periodic maps on Euclidean spaces without fixed points," Bulletin of the American Mathematical Society, vol. 67, pp. 471-474, 1961.

[4] P. E. Conner and E. E. Floyd, "On the construction of periodic maps without fixed points," Proceedings of the American Mathematical Society, vol. 10, pp. 354-360, 1959.

[5] A. L. Bas, "Some results on periodicity of difference equations," in Proceedings of the International Workshop Future Directions in Difference Equations, pp. 121-143, Vigo, Spain, June 2011.

[6] I. Györi and L. Horváth, "Widely applicable periodicity results for higher order difference equations," Journal of Difference Equations and Applications, 2013.

[7] I. Györi and L. Horváth, "Sharp algebraic periodicity conditions for linear higher order difference equations," Computers and Mathematics with Applications, vol. 64, no. 7, pp. 2262-22274, 2012.
[8] W. Rudin, Functional Analysis, McGraw-Hill, New York, NY, USA, 2nd edition, 1991.

[9] A. G. Ramm, "A simple proof of the Fredholm alternative and a characterization of the Fredholm operators," American Mathematical Monthly, vol. 108, no. 9, pp. 855-860, 2001.

[10] S. Elaydi, An Introduction to Difference Equations, Springer, New York, NY, USA, 2005.

[11] I. Györi and L. Horváth, "A new view of the $l^{p}$-theory for a system of higher order difference equations," Computers and Mathematics with Applications, vol. 59, no. 8, pp. 2918-2932, 2010. 


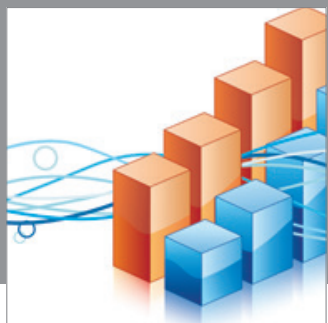

Advances in

Operations Research

mansans

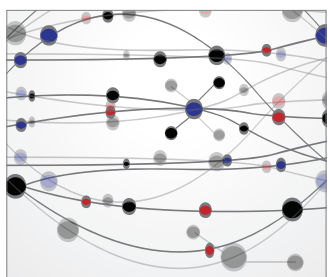

The Scientific World Journal
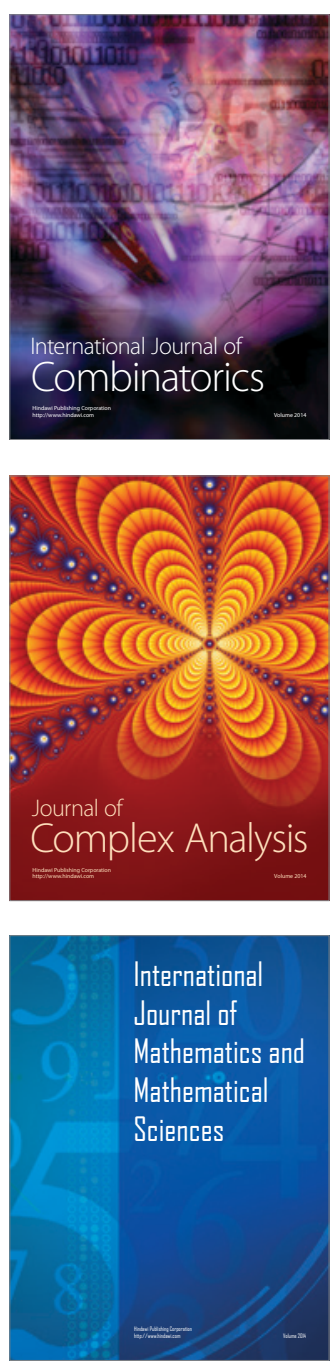
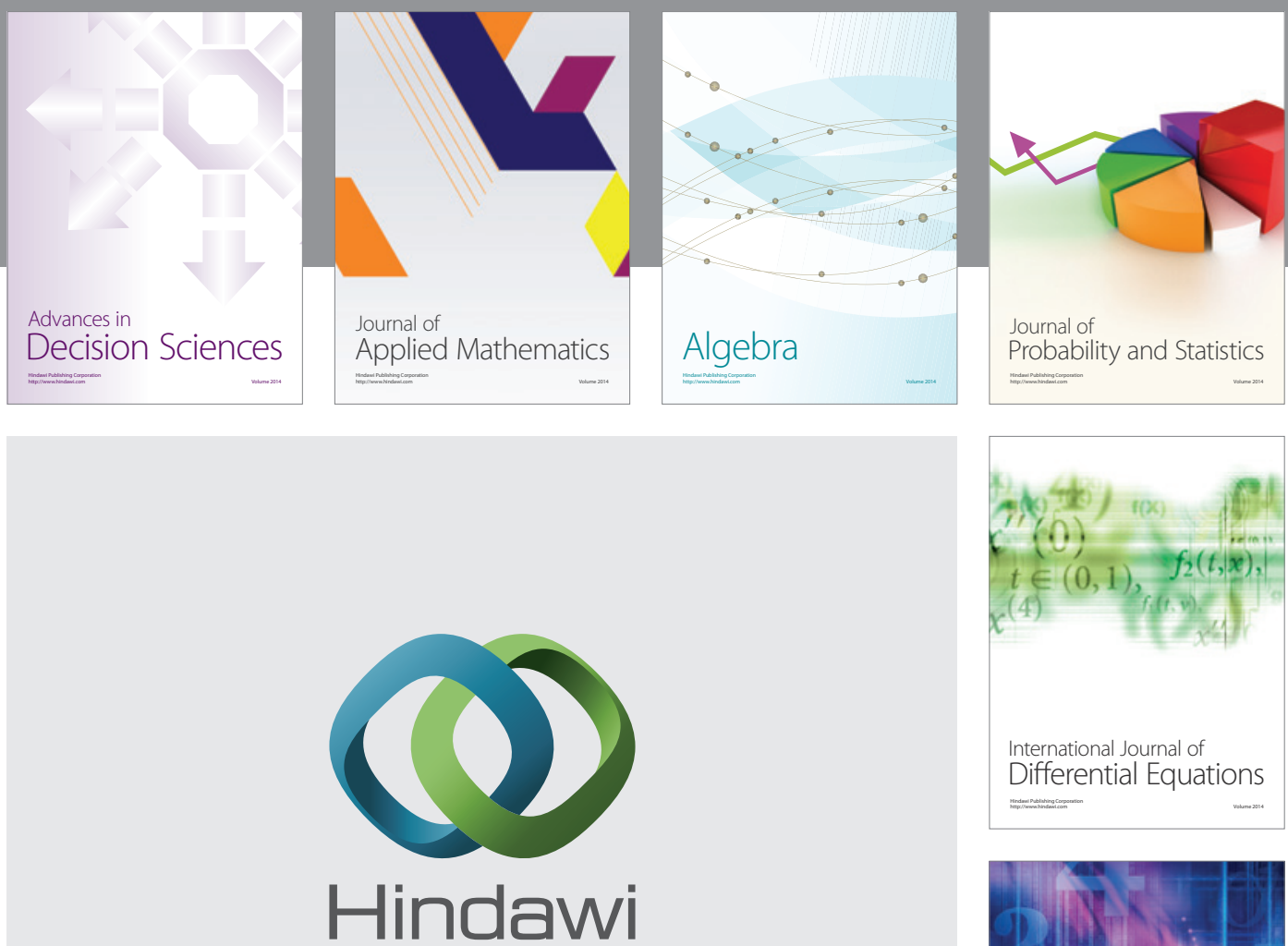

Submit your manuscripts at http://www.hindawi.com
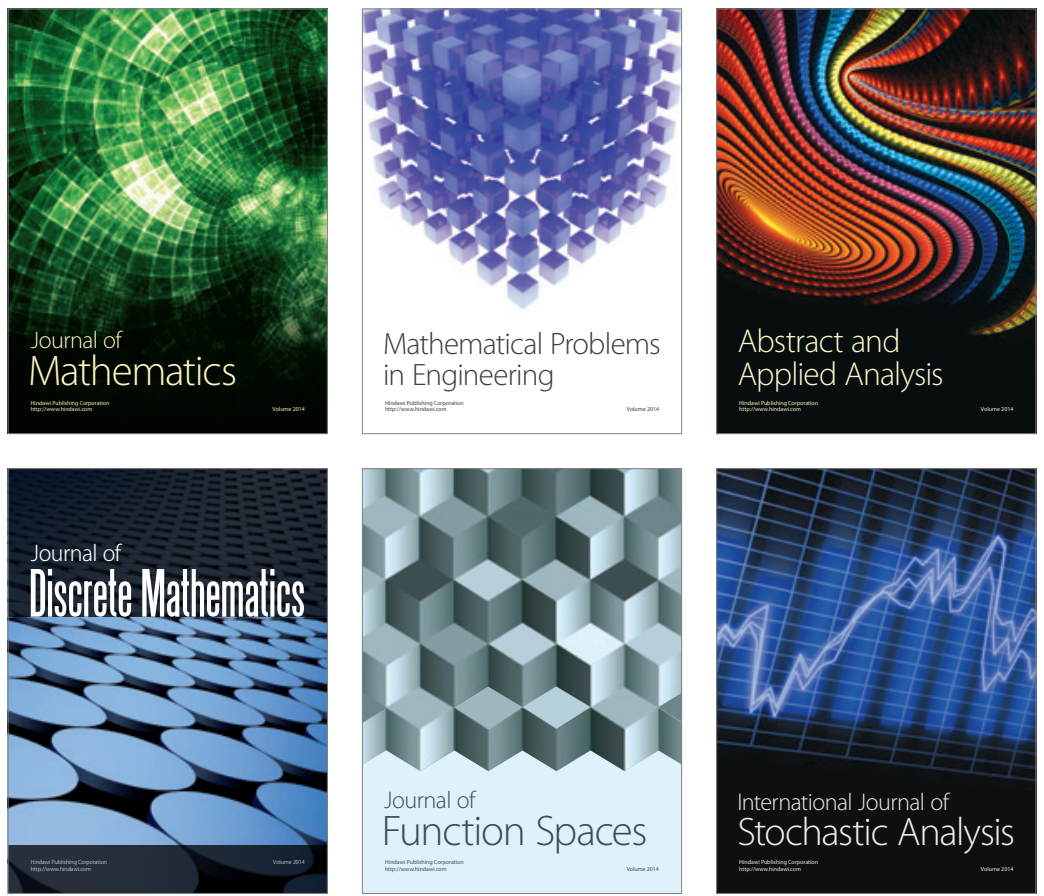

Journal of

Function Spaces

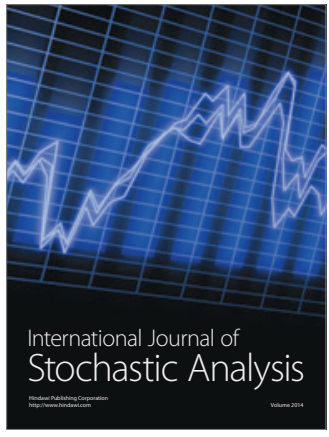

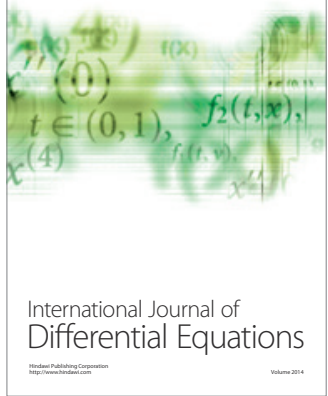
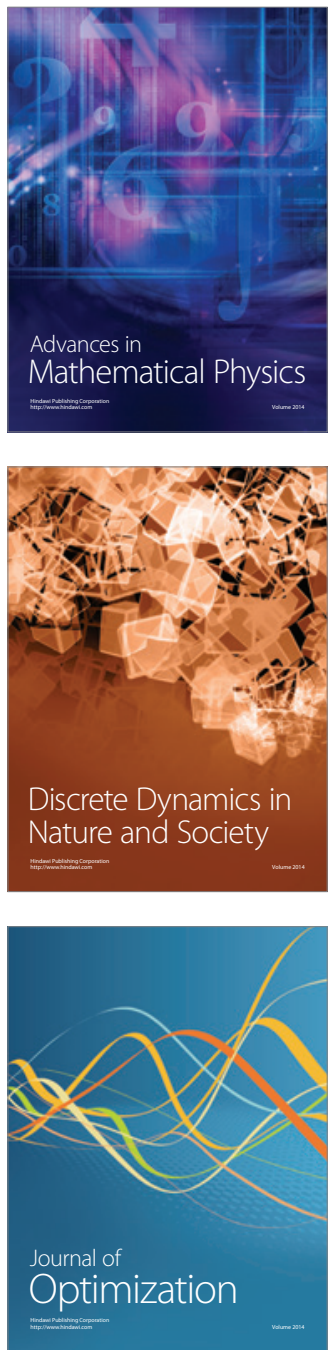